\section{CAR-T Cells: The Next Generation Cancer Therapy}

Received: September 24, 2018; Accepted: September 26, 2018; Published: October 02,2018

\section{Commentary}

Chimeric Antigen Receptor modified T cells (CAR- T) have undoubtedly become one of the epic inventions of science in recent years. Currently, there are two FDA approved CAR Therapies. Yescarta by Kite Pharmaceuticals for use in Diffuse Large B cell Lymphoma (DLBCL) [1] and Kymriah for use in relapsed Acute B- cell Lymphoblastic Leukemia (ALL) [2]. Both these therapies target CD19 positive B-cells to elicit cytotoxic response and ultimately kill the cancer cells. Both therapies have proven to be life saving for patients that were left with no choice after 2 or 3 relapses with chemotherapy and bone marrow transplant. Since their approval by FDA, many patient lives have been extended by a median of 12.9 months compared to 5 months by traditional chemotherapy [3]. Several other CAR therapies are now under scrutiny for treatment of multiple indications including Human Immunodeficiency Virus AIDS, Rheumatoid Arthritis and other solid tumors [4,5]. CAR technology is now exploring new strides by incorporating elements that confer enhanced T-cell functionality such as multisite recognition, gene editing CRISPR elements, co-signalling and co-stimulatory molecule. Why is that, the therapeutic with such potential to cure an incurable disease is practically unreachable to reach wider patient population. For one we have entered into an area of personalized medicine, where each CAR must be tailored from patients own immune cells, specifically $T$ cells. Cost and time to manufacture failures rates during manufacture and release testing, toxicities and side effects are few other causes. Kite pharmaceuticals announced that by its centralized manufacturing process, it has achieved a 99\% success rate with manufacturing CAR-T cells. In this process, T-cells are first collected from the patient in the hospital. These cells are then shipped off to a central manufacturing facility where they are genetically engineered to target the patient's cancer and expanded. Finally, they are shipped back to the hospital for infusion into the patient. Currently, the average manufacturing time is 17 days including the transportation of the material to and from the manufacturing facility to the patient care center. Research is also on-going to decrease turnaround time to engineer the isolated T-cells given the rapid progressive

\section{Shanthi Vadali* \\ St. Jude Children's Research Hospital - GMP LLC, Tennessee, USA}

*Corresponding author: Shanthi Vadali

shanthi.vadali@gmail.com

QC Scientist, St. Jude Children's Research Hospital, Tennessee, USA.

Tel: $1-337-302-4477$

Citation: Vadali S (2018) CAR-T Cells: The Next Generation Cancer Therapy. Arch Cancer Res. Vol.6 No.3:12

nature of this disease. Meanwhile, manufacturers of CAR-T cells are integrating automated production systems such as Miltenyi Biotec's CliniMACS Prodigy system that greatly decrease manual processing variability's and increase capacity in a GMP compliant system [6]. Other factors to consider are the severity of the patient disease, viability and strength of the isolated T cells to endure the engineering and expansion process. This is important because the severe the patient disease, a higher number of tumor cells are mixed in with the leukocytes, which makes the isolation of $T$ cells harder, increasing the risk of failure. Thus, it is important and relevant to look at the CAR-T therapy at an earlier stage of disease progression than as a last resort. Clinically, almost 1 out of every 5 patients exhibit mild to severe Cytokine Release Syndrome (CRS) when administered with CAR. Neurotoxicity is another concern with the administration of CAR. Per guidelines of FDA, hospitals are required to participate in the Risk evaluation and mitigation strategy program (REMS) and train a specialized team of nurses and doctors to handle the CRS response. In addition, the hospital stays, the remission rates of the patient, the follow up period add up to the heavy price tag of this valuable cure [7]. Nevertheless, there is a new hope for the patients that are suffering with this chronic illness and the hospitals, manufactures and CMC are trying to bring this therapy into patient reach.

\section{Conflict of Interest}

Authors declared that they had no conflict of interest. 


\section{References}

1 https://www.gilead.com/news/press-releases/2017/10/kitesyescarta-axicabtagene-ciloleucel-becomes-first-car-t-therapyapproved-by-the-fda-for-the-treatment-of-adult-patients-withrelapsed-or-refractory-large-bcell-lymphoma-after-two-or-morelines-of-systemic-therapy.

2 https://www.novartis.com/news/media-releases/novartis-receivesfirst-ever-fda-approval-car-t-cell-therapy-kymriahtm-ctl019.

3 https://www.medpagetoday.com/hematologyoncology/ leukemia/70917.
4 https://clinicaltrials.gov/ct2/results?cond=\&term=car\&cntry=\&stat $\mathrm{e}=\&$ city $=\&$ dist $=$.

5 Sadelain M, Brentjens R, Riviere I (2013) The basic principles of chimeric antigen receptor (CAR) design. Cancer Discov 3: 388-398.

6 https://www.bioprocessonline.com/doc/keys-to-scale-up-car-t-celltherapy-manufacturing-0001.

7 Cavallo J (2018) Weighing the cost and value of CAR T-cell therapy. A round table discussion with June $\mathrm{CH}$, Lonial S, Maloney DG and Touchon P. The ASCO Post. 